\title{
MULTILATERAL EXPLOITATION OF OLIVE TREE LOP - TECHNO-ECONOMICAL ANALYSIS FOR LEAVE EXTRACTS, COMPOST AND ENERGY PELLET PRODUCTION
}

\author{
P.P. Konstantakou ${ }^{1}$ \\ S.A. Haroutounian ${ }^{2}$ \\ V.G. Papadakis ${ }^{1}$ \\ ${ }^{I}$ University of Western Greece, Department of Environmental \& Natural \\ Resources Management, Agrinio, Greece \\ ${ }^{2}$ Agricultural University of Athens, Department of Science, Laboratory of \\ General Chemistry, Athens, Greece
}

\begin{abstract}
Agricultural residues in Greece are amounted to several million tons per year, the olive tree lops (OTL) ranked first with 1.4-3 million tons per year. After the removal of the thicker woods, the rest are burned in the countryside by farmers causing except from energy loss, particularly severe air pollution (greenhouse gases, micro particles, etc.). First objective of this study was to perform a techno-economical analysis of a complete management of OTL in a rural municipality (real application to the Municipality Department of Vasilaki in the Prefecture Laconia, Greece) for the final production of: a) leaves from OTL for extraction of useful constituents, b) bio-compost for soil enhancement and fertilizer and c) pellets for energy applications. From a sensitivity analysis, it was observed that the proposed investment was sensitive in changes of leaves value. Moreover, a change in the price of raw material alters considerably the investment. Generally, the expected profit was considered sufficient and the evaluation criteria (rate on return on initial investment, payout period and net present value) offer very promising results in the direction of undertaking the investment.

As the main result, this work envisages to opening up a new road showing a feasible solution of this problem, better than that used nowadays. The proposed solution could be extended to any agricultural residue and can be applied by any interested farmer, enterprise or local authority, offering exploitation of natural resources and enhancement of agriculturist income, especially those in less favored regions.
\end{abstract}

\section{KEYWORDS}

Olive Tree Lops (OTL); Oleuropein; Agricultural residues; Compost; Hydroxytyrosol; Olive Leaf Extract (OLE); Bioenergy; Pellet production.

\section{INTRODUCTION}

https://doi.org/10.15626/Eco-Tech.2010.066 
Organic wastes from animal production and agriculture and byproducts of agricultural and food processing industries have become major sources of environmental and social problems throughout the world in both developed and developing countries. Agricultural residues in Greece are amounted to several million tones per year, the olive tree lops (OTL) ranked first with 1.4-3 million tones per year. The management of these residues is crucial for the preservation of the environment and the valorization of these by-products. The most significant wastes are released by olive tree lops, as Greece is the third largest olive oil producer in the world. Olive tree lops are one of the most abundant agricultural organic residues in Greece, and the majority of them is burned immediately after harvesting the olive fruit and tree pruning operations (between November and March). This process can cause not only energy loss, but also particularly severe air pollution. Due to all these amount of OTL and the lack of planning and dissemination of their exploitation, the problem remains unsolved, constituting one of the most serious obstacle to sustainability of agriculture cultivation. It is estimated that from the 150 million olive trees in Greece, an amount of 3 million tones of OTL is produced annually, releasing by burning about 3.3 million tones $\mathrm{CO}_{2}$ [1-4]. As the calorific value of OTL is $3,500 \mathrm{kcal} / \mathrm{kg}$, these amounts could produce $12.2 \mathrm{TWh}$, sufficient to cover even the $20 \%$ of the total country's energy consumption. This enormous quantity of olive tree lops, that each year are produced in Greece, encourage their exploitation for the covering of various types of needs.

Thus, the main objective of this work is to demonstrate ways of exploitation of olive tree lopping instead of burning them in-situ, converting them to innovative and high-added value products, following a logic chain of exploitation: Removal of olive leaves (OL) for extraction of useful constituents (for pharmaceutical purposes or for cosmetics) or for animal feeding Composting of small branches to create an organic fertilizer - Conversion of the remaining quantities to pellets for energy production. Olive leaves contain several phenolic compounds and derivatives such as oleuropein, tyrosol and hydroxytyrosol (3,4-dihydroxyphenylenediamine). Nowadays, natural olive leaves and olive leaves extracts (OLE), are marketed as anti-aging, immunostimulators, and even antibiotics. Bioassays support its antibacterial, antifungal, and anti-inflammatory effects at a laboratory level. A liquid extract made directly from fresh olive leaves recently gained international attention, when it was shown to have an antioxidant capacity almost double green tea extract and $400 \%$ higher than Vitamin C. Because of their beneficent quality, the price of olive leaves is approximately $2 € / \mathrm{kg}$. Equally important may be the solution of the conversion of olive tree lops in biomass and energy exploitation. Biomass can be used as fuel for energy production via intermediate products such as bio-ethanol, biogas, methanol, pellets, etc. Emissions such as NOx, SOx and volatile organic compounds from pellet burning equipment, are, in general, very low in comparison to other forms of combustion heating, making this one of the less-polluting heating options available.

Furthermore, composting process which performed during this work, offered a product very rich in nutritious components for plants and useful for many purposes. Plastic buckets were used for the composting of the OTL, which were first crumbled, so that being easier the process of their deconstruction. During decomposition time, the variation and the behavior of parameters such as temperature, humidity and oxygen content in the buckets were studied, and furthermore the appropriateness of the mature compost for the plants. The addition of other agricultural and horticultural residues was also studied for co-composting. A schematic representation of a rational procedure for OTL exploitation is suggested below, Figures 1-4: 
Linnaeus ECO-TECH '10

Kalmar, Sweden, November 22-24, 2010

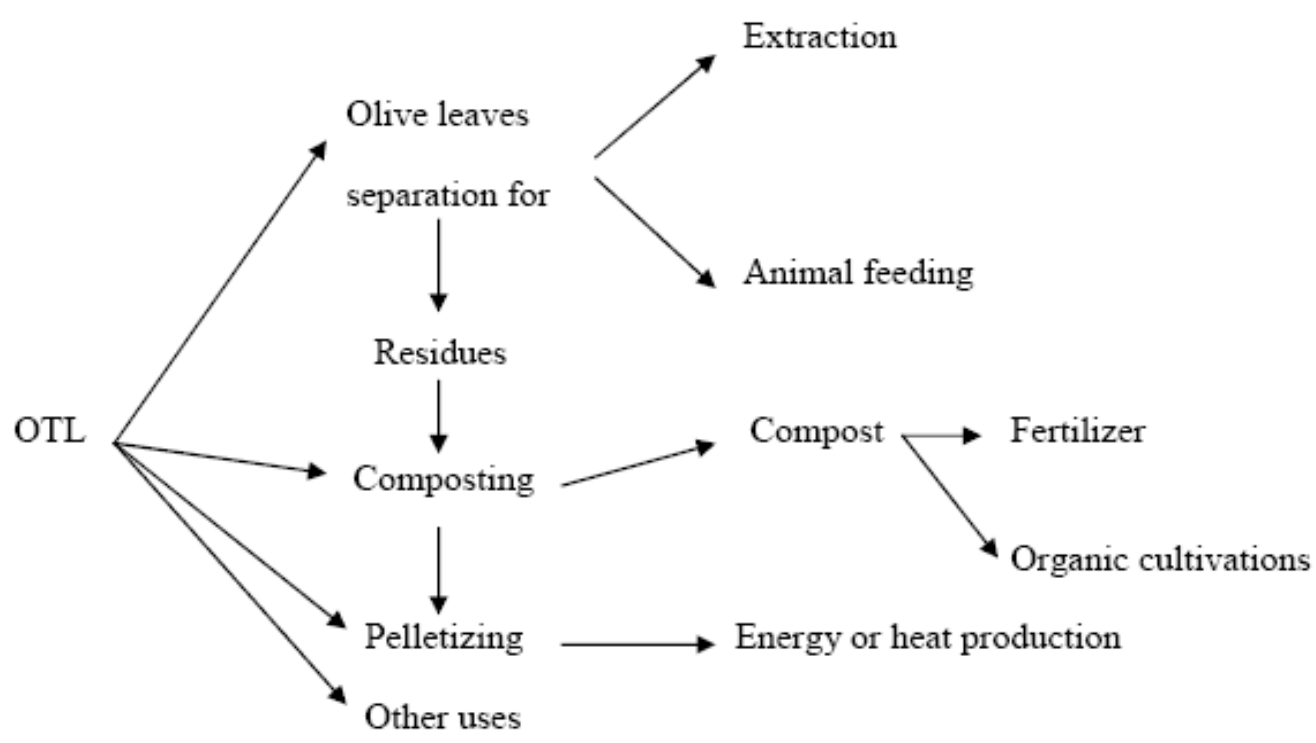

Figure 1. Process diagram of OTL exploitation methods

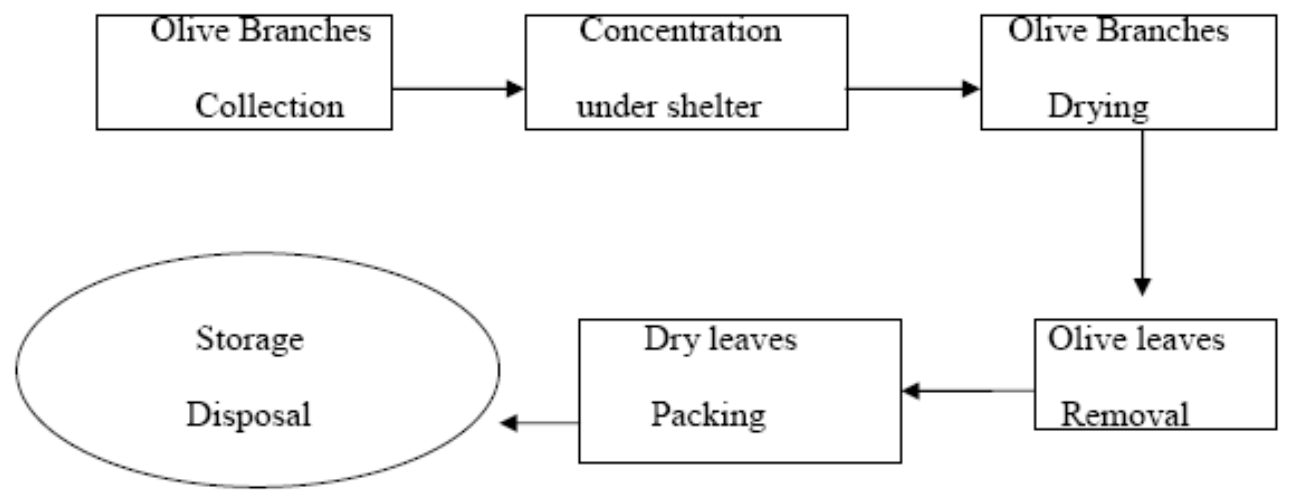

Figure 2. Olive leaves removal

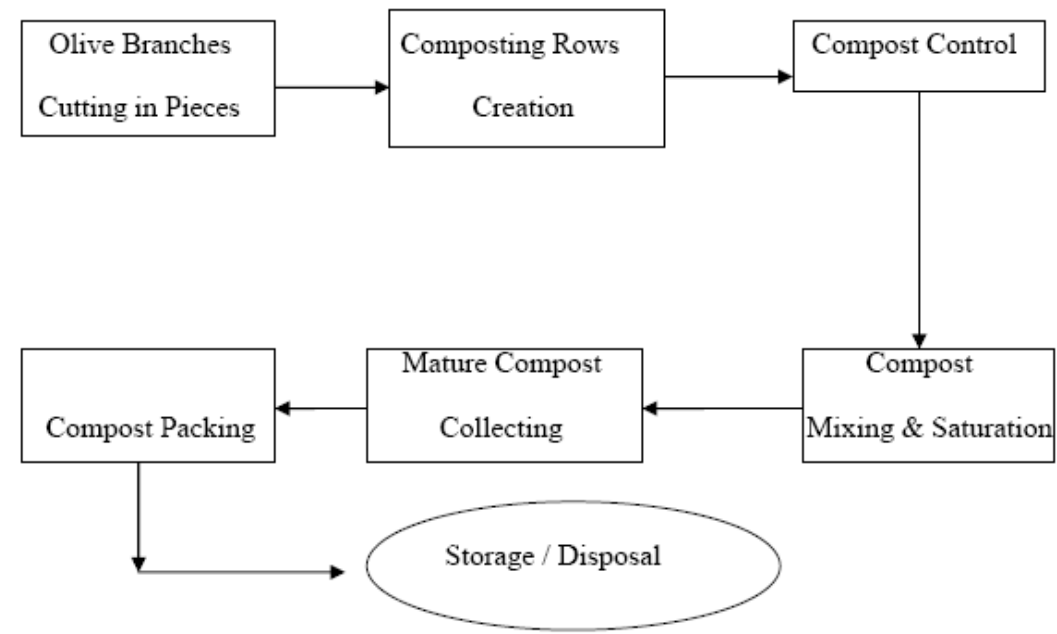

Figure 3. Olive tree lops composting 


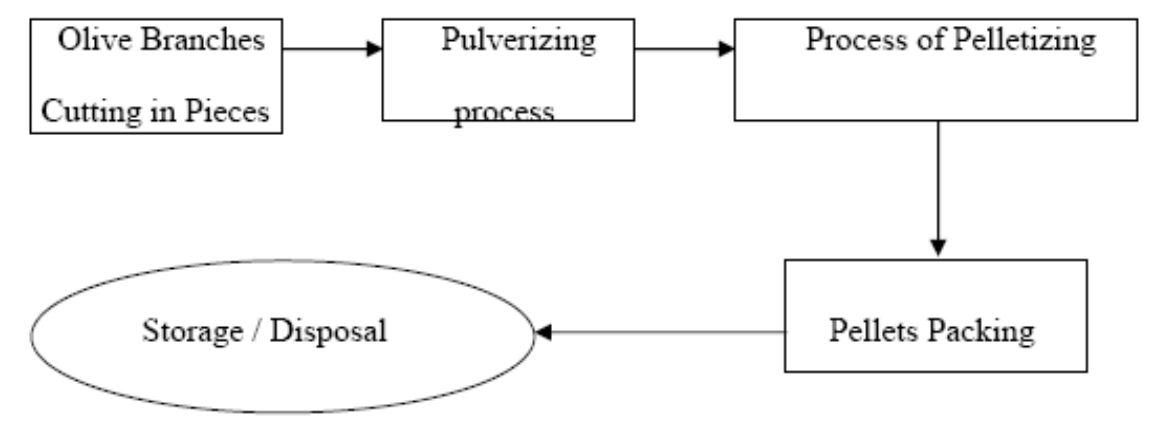

Figure 4. Olive tree lops conversion to energy pellets

\section{EXPERIMENTAL STUDY OF OTL COMPOSTING}

\subsection{Composting process}

The exploitation process presented in this study was the compost production. Composting has been shown to be a suitable method for exploiting the huge quantity of olive tree lops for the production of a high quality compost. The composting process that implemented was the type of "home composting". The process was carried out in two plastic buckets of 4001t and 6001t. The raw material which used for the composting process was mainly OTL, occasionally using for co-composting grass and green domestic wastes (GDW). The addition of grass into the mixture can perfectly correct the $\mathrm{C} / \mathrm{N}$ ratio to an optimum value of 30:1 and offering a mixing of the different feedstock materials. No specific chemical was used as activator; only pigeon dropping (PD). The composting process started during the summer and the stable and mature compost was produced after approximately four months (June - September). Prior to experimentation, OTLs were cut into small pieces less than $1 \mathrm{~cm}$ in lenth for their faster degradation. The 4001t plastic bucker was filled with OTL and grass and the 6001t one was filled with OTL, grass and green domestic wastes. The aim of that experiment was to determine the basic quality differences between two final composts, their composting process and the completion time for each of them. The composting process requires adequate conditions of $\mathrm{pH}$, temperature, moisture, oxygenation and nutrients, to allow the adequate development of the microbial population [4].

A week after the initiation of the process, the material mass was appreciably decreased. The buckers were filled again, so that it is possible for the temperature to get to the necessary for the process levels. Once a week the two composts were watered in order to obtain a suitable humidity. Water was added regularly to avoid the humidity level to drop below $40 \%$. After watering, a turning of the compost volume was done aiming at its complete aeration and to obtain an oxygen content above $10 \%$. Since the initiation day, temperature, moisture and oxygen levels were measured regularly, every week. 

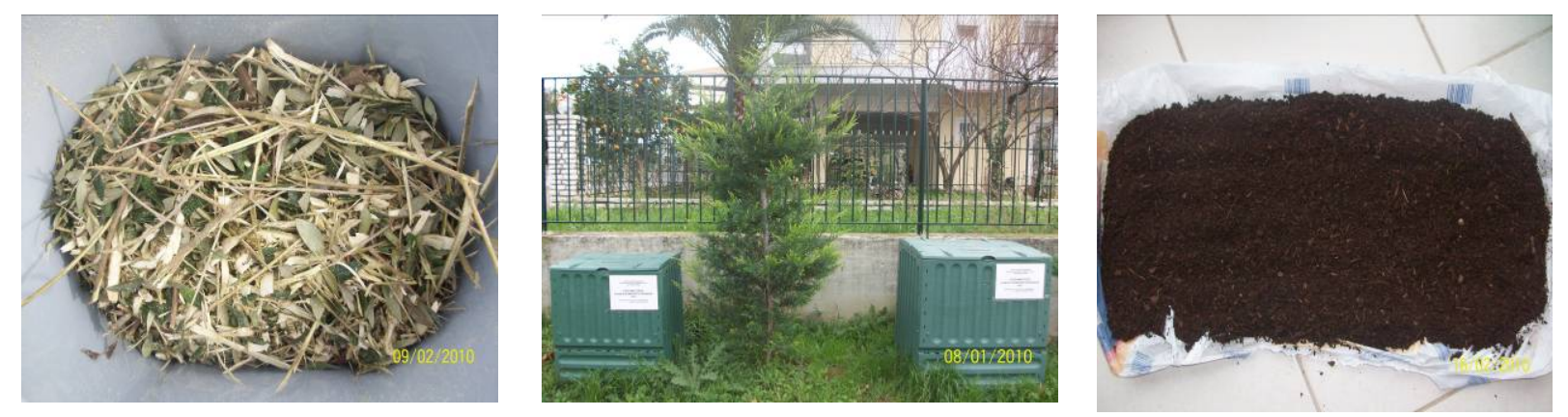

Figure 5. Experimental composting buckets (middle) converting fresh OTL (left) to mature compost (right).

\subsection{Sample analyses}

\subsubsection{Physicochemical methods}

Temperature in the core of each bucket was monitored on a daily basis. Turning was mostly performed weekly, but temperature fluctuations were also taken into account and turning occurred more frequently when the temperature increased above $65{ }^{\circ} \mathrm{C}$ or declined below 55 ${ }^{\circ} \mathrm{C}$. During process, a series of physicochemical parameters were measured, including:

- Moisture content, determined by drying a sample at $105{ }^{\circ} \mathrm{C}$ until observations of constant weight.

- $\mathrm{pH}$ and electrical conductivity (EC) in water extract, by diluting 1 part of compost by volume, with 1.5 parts of distilled water [2].

- Total nitrogen, by using the micro-Kjeldahl method [3].

- Concentration of total P, determined with an atomic absorption spectrophotometer [4].

\subsubsection{Agronomic evaluation method}

The agronomic value of the composted products was tested by the following procedure. The mature and stable sample of compost was riddled by a $6-7 \mathrm{~cm}$ sieve. Seeds of Lepidium Sativum remained in water for 8 hours [5]. Then, ten seeds of Lepidium Sativum were planted in compost sample. Into the three first days, seeds began planting. Lepidium Sativum leaves maintained itself greens and they did not yellow.

The green colour of the leaves was the proof both for stability and maturity of final composts, indication also of a non-phytotoxicity in these [6].

\subsection{Results and discussion}

Temperature during the first week arose at a maximum value and then was decreased gradually until stabilization. It was also observed that the increase of temperature caused a reduction on the compost's oxygen content. That was owed to the microbial action which is much accountable when temperature increases.

The moisture content in the buckets tended to increase throughout the composting process reaching high values, perhaps because of the important grass or GDW quantity and their high 
water content. The moisture of the final composts was about $30 \%$. pH values were between 6.5 -7.5 during all the composting process. The $\mathrm{pH}$ of final composts was 8.0 for the compost originated from OTL and grass and 7.9 for the compost from OTL, grass and green domestic wastes. The total phosphorus concentration for the final composts was $6.8 \%$ and $6.2 \%$, respectively.

Table 1. Operational parameters during the composting process for the 400lt bucket

\begin{tabular}{cccc}
\hline $\begin{array}{c}\text { Composting } \\
\text { time (days) }\end{array}$ & $\mathrm{T}\left({ }^{\circ} \mathrm{C}\right)$ & $\mathrm{O}_{2}(\%)$ & $\mathrm{RH}(\%)$ \\
\hline 1 & 36.1 & 18.3 & 85.1 \\
\hline 15 & 59.7 & 15.5 & 75.5 \\
\hline 30 & 45.3 & 16.2 & 67.3 \\
\hline 45 & 41.2 & 16.5 & 72.4 \\
\hline 60 & 35.4 & 17.1 & 71.0 \\
\hline 75 & 28.3 & 18.1 & 73.6 \\
\hline 90 & 27.9 & 18.3 & 76.9 \\
\hline
\end{tabular}

Table 2. Operational parameters during the composting process for the 600lt bucket

\begin{tabular}{cccc}
\hline $\begin{array}{c}\text { Composting } \\
\text { time (days) }\end{array}$ & $\mathrm{T}\left({ }^{\circ} \mathrm{C}\right)$ & $\mathrm{O}_{2}(\%)$ & $\mathrm{RH}(\%)$ \\
\hline 1 & 36.6 & 18.4 & 82.8 \\
\hline 15 & 52.3 & 17.7 & 100 \\
\hline 30 & 58.1 & 15.7 & 92.4 \\
\hline 45 & 45.8 & 15.3 & 79.2 \\
\hline 60 & 39.2 & 16.1 & 80.3 \\
\hline 75 & 33.9 & 18.3 & 75.1 \\
\hline 90 & 31.7 & 19.8 & 73.2 \\
\hline
\end{tabular}

\section{TECHNO-ECONOMICAL ANALYSES}

The main objective of the present work is to perform a techno-economical study, for design and implementation of a pilot plant for an integrated management of OTL in the Municipal Department of Basilaki, Prefecture of Laconia, Greece. This area counts approximately 300,000 olive trees. Besides, in average these olive trees produce 2,100 t/yr of olives that milled producing $420 \mathrm{t} / \mathrm{yr}$ olive oil. The quantity of OTL produced from the harvesting and pruning is estimated on 6,000 $\mathrm{t} / \mathrm{yr}$ on average; this is the designed capacity for the unit under consideration. Three final products are envisaged: a) separate olive leaves for disposal to pharmaceutical or cosmetics industry, b) compost for agriculture and soil enhancement and c) pellets for energy applications. In this techno-economical analysis the dependence of the rate of the return on investment (ROI) on various important characteristics was estimated, suggesting the minimum percentage of leaves required for break-even point (436 t OL / 6000 t OTL i.e., $7 \%$ of the total OTL), and also indicating the sensitivity on raw material value and on fixed cost value, see Figures $6 \& 7$. 


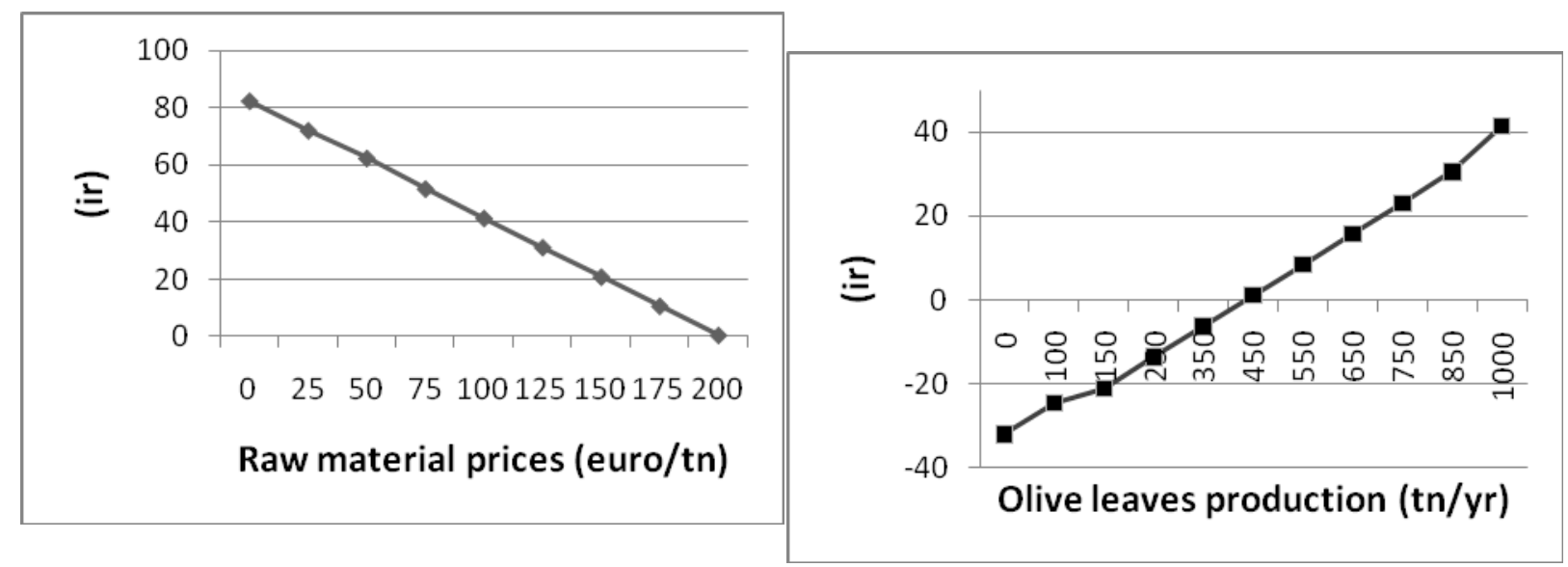

Figure 6. Dependence of the rate of return on investment, $i_{r}(\%)$, from the raw material value (left) and from the olive leaves production (right).

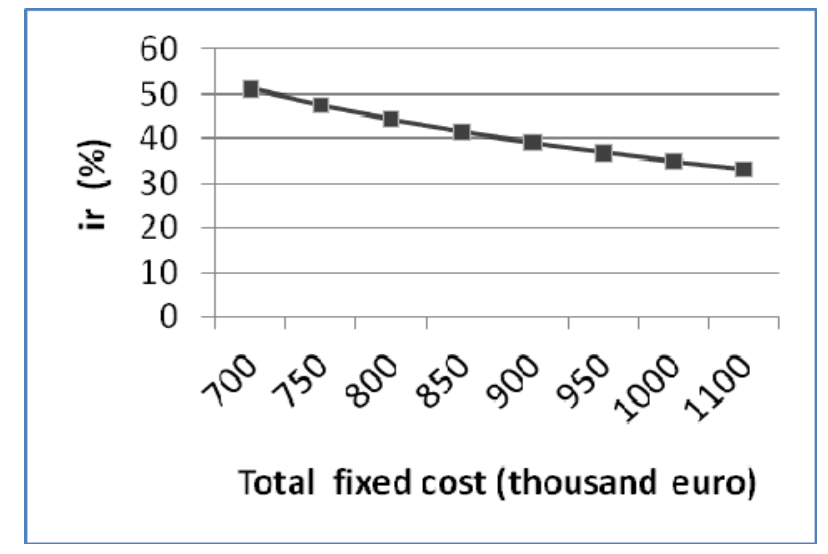

Figure 7. Dependence of the rate of return on investment, $i_{r}$, from the fixed cost value.

\section{CONCLUSIONS}

It was clearly observed from the sensitivity results of the techno-economical analysis that the proposed investment on OTL complete exploitation is very sensitive on the production and disposal of olive leaves. A minimum level of 7\% of olive leaves production from all OTL is required in order to ensure viability. The other parameters presented herein have the expected influence on ROI; the higher the raw materials price and the higher the fixed cost value, the lower the ROI observed, with a more significant dependence from the raw material price. In general, however, it could be stated with certainty that the criteria of ROI, payout period and net present value (NPV), by taking into consideration the above sensitivity results, the presented OTL exploitation is suggested to be undertaken.

Additionally, composting process presented shortly in this work indicates that OTL is an excellent raw material for composting producing a high quality compost. Composting process during summer time is efficient enough, as it is assisted by the environmental conditions.

The present work, as a general concept, contributes to opening up a paved road showing a feasible solution of the problem of OTL management. The proposed solution could be further extended to any agricultural residue offering multiple environmental and economical benefits. 


\section{ACKNOWLEDGMENTS}

Authors wish to thank the European Commission for financial support for dissemination of their results under FP7-Regions-2009-1, SWAM project (245427) and STInno project (245405).

\section{REFERENCES}

[1] Gil, M.V., Carballo, M.T., Calvo, L.F., 2008. Fertilization of maize with compost from cattle manure supplemented with additional mineral nutrients, Waste Management 28, 14321440 .

[2] Zmora-Nahum, S., Markovitch, O., Tarchitzky, J., Chen, Y., 2005. Dissolved organic carbon (DOC) as a parameter of compost maturity, Soil Biology \& Biochemistry 37, 21092116.

[3] Manios, T., 2004. The composting potential of different organic solid wastes: experience from the island of Crete, Environment International 29, 1079- 1089.

[4] Sellami, F., Jarboui, R., Hachicha, S., Medhioub, K., Ammar, E., 2008. Co-composting of oil exhausted olive-cake, poultry manure and industrial residues of agro-food activity for soil amendment, Bioresource Technology 99, 1177-1188.

[5] Garcia-Gomez, A., Roig, A., Bernal, M.P., 2003. Composting of the solid fraction of olive mill wastewater with olive leaves: organic matter degradation and biological activity, Bioresource Technology 86, 59-64.

[6] Komilis, D.P., Tziouvaras, I.S., 2009. A statistical analysis to assess the maturity and stability of six composts. Waste management $29,1504-1513$. 